\title{
Coincident Electron Channeling and Cathodoluminescence Studies of Threading Dislocations in GaN
}

\author{
Gunasekar Naresh-Kumar, ${ }^{1, \star}$ Jochen Bruckbauer, ${ }^{1}$ Paul R. Edwards, ${ }^{1}$ Simon Kraeusel, ${ }^{1}$ \\ Ben Hourahine, ${ }^{1}$ Robert W. Martin, ${ }^{1}$ Menno J. Kappers, ${ }^{2}$ Michelle A. Moram, ${ }^{2,3}$ \\ Stephen Lovelock, ${ }^{2}$ Rachel A. Oliver, ${ }^{2}$ Colin J. Humphreys, ${ }^{2}$ and Carol Trager-Cowan ${ }^{1}$ \\ ${ }^{1}$ Department of Physics, SUPA, University of Strathclyde, Glasgow G4 ONG, UK \\ ${ }^{2}$ Department of Materials Science and Metallurgy, University of Cambridge, Cambridge CB2 3QZ, UK \\ ${ }^{3}$ Department of Materials, Imperial College London, Exhibition Road, London SW7 2AZ, UK
}

\begin{abstract}
We combine two scanning electron microscopy techniques to investigate the influence of dislocations on the light emission from nitride semiconductors. Combining electron channeling contrast imaging and cathodoluminescence imaging enables both the structural and luminescence properties of a sample to be investigated without structural damage to the sample. The electron channeling contrast image is very sensitive to distortions of the crystal lattice, resulting in individual threading dislocations appearing as spots with black-white contrast. Dislocations giving rise to nonradiative recombination are observed as black spots in the cathodoluminescence image. Comparison of the images from exactly the same micron-scale region of a sample demonstrates a one-to-one correlation between the presence of single threading dislocations and resolved dark spots in the cathodoluminescence image. In addition, we have also obtained an atomic force microscopy image from the same region of the sample, which confirms that both pure edge dislocations and those with a screw component (i.e., screw and mixed dislocations) act as nonradiative recombination centers for the Si-doped $c$-plane GaN thin film investigated.
\end{abstract}

Key words: electron channeling, cathodoluminescence, SEM, diffraction, spectroscopy, nitrides, dislocations

\section{INTRODUCTION}

In the last two decades, GaN and its alloys with AlN and InN have revolutionized the field of ultraviolet (UV), blue, and green light-emitting diodes (LEDs) and laser diodes. Such nitride-based devices operate in spite of exhibiting threading dislocation (TD) densities as high as $10^{10} \mathrm{~cm}^{-2}$ (Lester et al., 1995). However, the reduction of TD densities in nitride semiconductors has been shown to improve the quantum efficiency of the light emission for both InGaNand AlGaN-based structures, and it has been clearly demonstrated that TDs in nitrides give rise to nonradiative recombination (Karpov \& Makarov, 2002; Amano et al., 2004; Dai et al., 2009; Ban et al., 2011; Kneissl et al., 2011; Harada et al., 2012; Schiavon et al., 2013). One report does, however, show comparable internal quantum efficiency for blue-(but not UV)emitting InGaN/GaN LEDs grown on sapphire and on GaN despite a substantial difference in defect densities (Cao et al., 2004). There is still debate as to how TDs influence the light emission in nitride semiconductors. Most theory predicts that all TDs (edge, screw, or mixed) should lead to quenching of the luminescence either due to deep electronic states introduced into the band gap by the dislocation itself, or due to the segregation of vacancies, impurities, and/or dopants to the dislocation (Wright \& Grossner, 1998; Leung et al., 1999; Northrup, 2001, 2002;

Received June 3, 2013; accepted October 7, 2013

*Corresponding author. E-mail: naresh.gunasekar@strath.ac.uk
Lymperakis et al., 2004). The exception is the work of Elsner et al. (1997), who predicted that both screw and edge TDs are electrically inactive and thus would not quench the luminescence. There are very few publications reporting the correlation of TD type with luminescence behavior. Albrecht et al. (2008) combined cathodoluminescence (CL) with defect-selective etching studies and demonstrated that the presence of edge and mixed TDs in a GaN thin film grown by hydride vapor phase epitaxy led to nonradiative recombination. However, they also observed that screw dislocations did not influence the luminescence. Yamamoto et al. (2003) carried out CL in a transmission electron microscope to interrogate the properties of an epitaxial laterally overgrown InGaN/GaN multiple quantum well structure. They observed that edge and mixed dislocations running parallel to the $c$-axis led to nonradiative recombination. They also observed screw type TDs running parallel to the (0001) surface and found that these did not affect the luminescence.

An extensive investigation of the influence of individual TDs on the luminescence properties of nitride semiconductors grown under a range of different growth conditions has yet to be undertaken, as the techniques available to date are time consuming and require great care to prepare suitable samples. In this article we describe a method, combining electron channeling contrast imaging (ECCI) and CL imaging in a field emission scanning electron microscope (SEM), with which it is possible to rapidly and nondestructively obtain coincident structural and luminescence data 
from individual TDs over large areas $\left(>100 \mu \mathrm{m}^{2}\right)$ of a sample. We report preliminary results from a Si-doped $c$-plane GaN thin film. The results obtained from ECCI-CL were also compared to those obtained with atomic force microscopy (AFM) data from exactly the same region of the sample.

\section{Materials and Methods}

ECCI is an emerging technique for characterizing extended defects in nitride semiconductors, where images are produced by electrons that channel down the crystal planes (Trager-Cowan et al., 2007; Picard et al., 2007; NareshKumar et al., 2012a, 2012b). Extremely small changes in crystallographic orientation and local strain are revealed by changes in contrast in a channeling image constructed by monitoring the intensity of backscattered electrons as the electron beam is scanned over a suitably oriented sample. Low angle tilt and rotation boundaries, atomic steps, and dislocations are thus observed in an electron channeling contrast (ECC) image. TDs appear as spots with blackwhite contrast in the ECC image. For materials with a wurtzite crystal structure (such as $\mathrm{GaN}$ ), we have developed a simple geometric procedure to identify a given TD as edge, screw, or mixed by exploiting differences in the direction of the black-white contrast between two ECC images acquired with symmetric channeling conditions (NareshKumar et al., 2012a).

The conditions required to resolve individual TDs in an ECC image are quite stringent: a high brightness, small (nanometers), high current (nanoamps or higher), low divergence (a few mrad) electron beam, is required. Such conditions are met in a field emission SEM. ECC images are generally acquired at electron beam energies of $20-30 \mathrm{keV}$ to obtain the best signal-to-noise ratio. The performance of the diodes used to detect backscattered electrons reduces for lower electron beam energies (Wilkinson \& Hirsch, 1997). The spatial and depth resolution is of the order of tens of nanometers.

$\mathrm{CL}$, the emission of light when a material is bombarded by an electron beam, is well established as a spectroscopic characterization tool in the study of light-emitting semiconductors. CL spectra may provide information on composition, strain, defects, and doping of a sample, as exemplified in Yacobi and Holt (1990) and Parish and Russell (2007). CL can be used to obtain both spectrally and spatially resolved luminescence properties of materials and is particularly attractive as it is possible to confine the injection of charge carriers within a substantially sub- $\mu \mathrm{m}$-scale volume. For example, using a field emission SEM and limiting the excitation volume by selecting a low accelerating voltage, $20 \mathrm{~nm}$ spaced QWs in GaAs have been resolved (Norman, 2000), while peak shifts in the emission from InGaN/GaN quantum wells over an $\approx 10 \mathrm{~nm}$ distance have been measured (Bruckbauer et al., 2011; Edwards \& Martin, 2011). A major challenge in using low accelerating voltages, particularly when coupled with the low beam current required for a small spot size, is in detecting the resulting much-reduced light intensity. Therefore, in any measurement, a compromise has to be made between resolution and the signal-to-noise ratio. TDs which give rise to nonradiative recombination are observed as dark spots in a CL image. If the excitation volume is sufficiently small, the size of the dark spots is determined by the minority carrier diffusion length (Yakimov, 2012).

The results presented in this article are from a GaN:Si sample grown using a Thomas Swan $6 \times 2$-inch closecoupled showerhead metal-organic vapor phase epitaxy (MOVPE) reactor. The precursors were trimethylgallium, silane $\left(\mathrm{SiH}_{4}\right)$, and ammonia $\left(\mathrm{NH}_{3}\right)$, with $\mathrm{H}_{2}$ as the carrier gas. (0001) orientated sapphire (c-plane) served as the substrate. A nominally $30 \mathrm{~nm}$ thick GaN nucleation layer was grown before a $5 \mu \mathrm{m}$ thick coalesced $\mathrm{GaN}$ layer, which formed after three-dimensional island growth. A multilayer was then grown containing four $n$-doped $200 \mathrm{~nm}$ thick $\mathrm{GaN}$ layers with increasing Si-doping concentration $(5.5 \times$ $10^{17}$ to $1.0 \times 10^{19} \mathrm{~cm}^{-3}$ ) separated by $200 \mathrm{~nm}$ thick undoped GaN spacer layers. Finally, a $200 \mathrm{~nm}$ thick GaN spacer layer followed by a $\mathrm{Si}$-doped $\mathrm{GaN}$ layer with a $\mathrm{Si}$ concentration of $1.0 \times 10^{18} \mathrm{~cm}^{-3}$ as the top layer were grown (Bakshi et al., 2009). For the results presented in this article, only the top $200 \mathrm{~nm}$ Si-doped GaN layer was probed.

Both ECC and CL imaging were performed using an FEI Sirion field emission SEM (FEI, Eindhoven, The Netherlands). Figure 1 shows the schematics of the experimental setups used for coincident acquisition of ECC and CL images. ECC images were acquired with an electron beam spot of $\approx 4 \mathrm{~nm}$, a beam current of $\approx 2.5 \mathrm{nA}$, a beam divergence of $\approx 4 \mathrm{mrad}$, and an electron beam energy of $30 \mathrm{keV}$. As depicted in Figure 1a, we used a forescatter geometry where the sample is tilted between 30 and $70^{\circ}$ to the impinging electron beam and the backscattered electrons are detected by an electron-sensitive diode placed in front of the sample (Trager-Cowan et al., 2007; Naresh-Kumar et al., 2012a, $2012 b$ ). It is also necessary to use a detection system that allows discrimination between electrons leaving the sample that carry channeling information and those that have been diffusely scattered by the sample. An amplifier that can offset the diffuse background signal and amplify the channeling signal is required. We used electron-sensitive diodes and a signal amplifier provided by K.E. Developments Ltd.

Room temperature CL images were acquired with an electron beam spot size of $\approx 17 \mathrm{~nm}$, a beam current of $\approx 6 \mathrm{nA}$, and an electron beam energy of $5 \mathrm{keV}$. Monte Carlo simulations (Drouin et al., 2007) show that for a $5 \mathrm{keV}$ electron beam normally incident on a $\mathrm{GaN}$ surface, $90 \%$ of carriers are generated within a volume $<100 \mathrm{~nm}$ in diameter. As illustrated in Figure 1b, the sample was tilted at $45^{\circ}$ to the incident electron beam, and the luminescence was collected by a reflecting objective with its optical axis perpendicular to the electron beam and was detected using an Oriel MS125 spectrograph (Oriel Instruments, Stratford, CT, USA) with a cooled Andor Technology Newton electron multiplying charge-coupled device (Andor Technology, Belfast, N. Ireland, UK). A room temperature CL spectrum was 

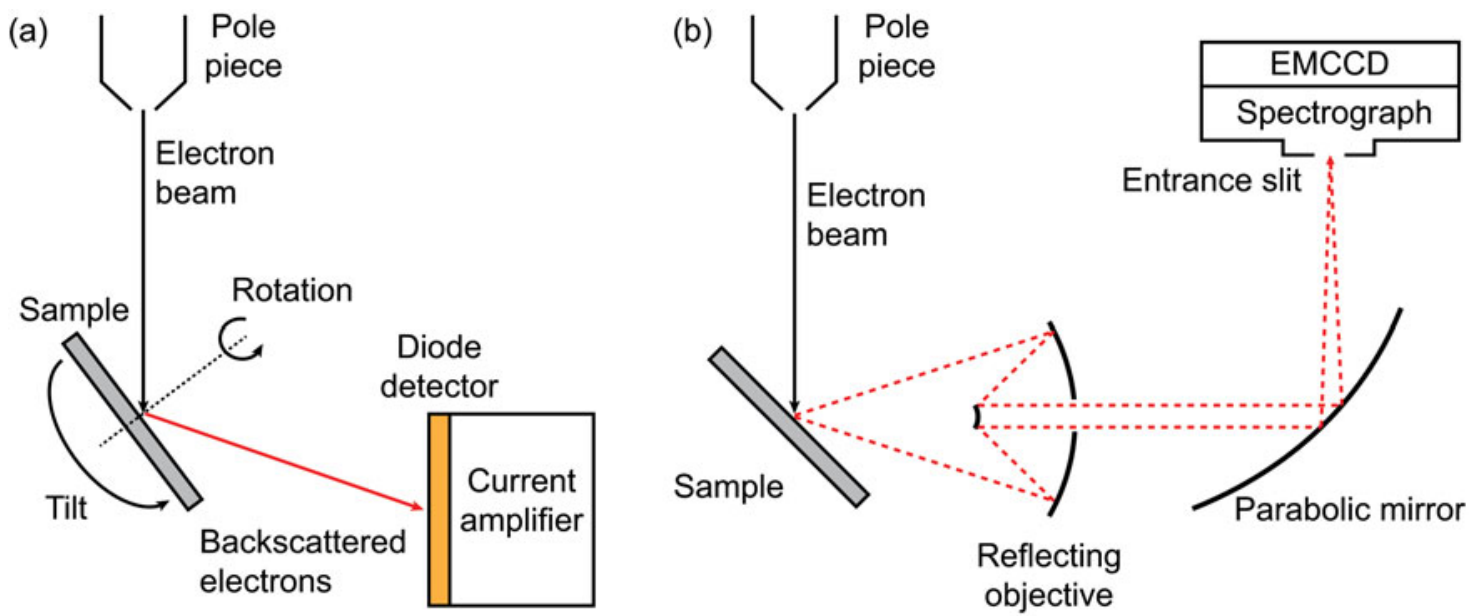

Figure 1. Schematics of the (a) electron channeling contrast imaging and (b) cathodoluminescence experimental setups.

acquired at each point on a raster as the electron beam was scanned across the sample surface. In the present work a raster step of $40 \mathrm{~nm}$ was used. After data acquisition, peaks were fitted to each constituent spectrum (a Voigt function was used in the present work), and the resulting fitting parameters (e.g., peak position, intensity, width, etc.) plotted to create two-dimensional images, which map variations in the emission characteristics. A detailed description of the hyperspectral CL system can be found in Martin et al. (2004) and Edwards et al. (2012).

Finally, we compared our ECCI and CL results with those obtained by AFM. Tapping mode AFM images were acquired using a Digital Instruments Dimension 3100 microscope (Digital Instruments, now Veeco, Santa Barbara, CA, USA) employing Bruker-Nano RTESP probes (Bruker Nano Inc., Santa Barbara, CA, USA) with a nominal end radius of $8 \mathrm{~nm}$.

TDs form small pits when they terminate at the sample surface and this change in the topography can be detected by AFM. In the present work the sample surface was subjected to an in situ treatment using $\mathrm{SiH}_{4}$ and $\mathrm{NH}_{3}$ immediately after growth in the MOVPE reactor, which enlarges the size of the pits and makes it easier to detect dislocations using AFM. In addition, the pit size may be used to distinguish edge type TDs from those with a screw component (i.e., screw and mixed dislocations): the pit size for an edge dislocation is smaller than those with a screw component (Oliver et al., 2006b).

\section{Results AND Discussion}

Figure 2 shows a typical ECC image from this sample. The differences in gray scale reveal regions of different tilt or twist across the sample and as discussed above, TDs appear as spots with black-white contrast. Note that channeling contrast is still clearly observed from the film in spite of the $\mathrm{SiH}_{4}$ treatment of the surface. For this image it is possible to discriminate between TDs, which are further than $\approx 150 \mathrm{~nm}$

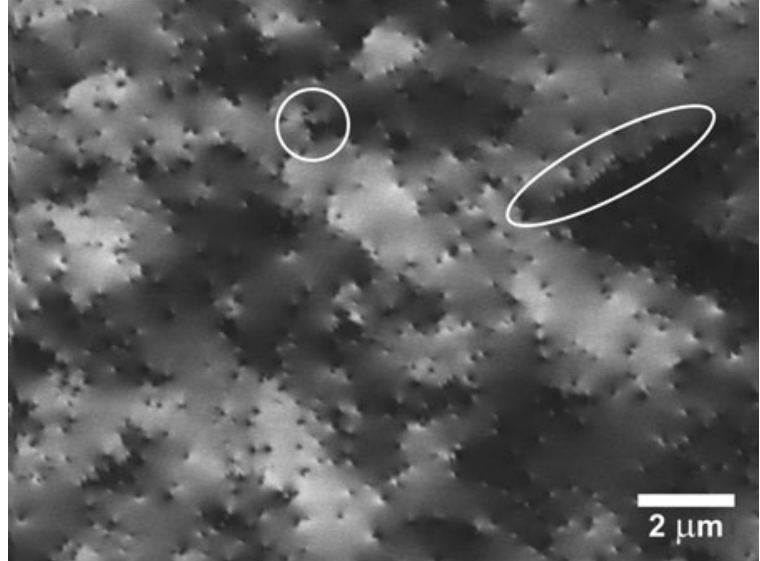

Figure 2. Typical electron channeling contrast image showing threading dislocations (TDs) and tilt/twist across the sample. The white ellipse highlights a line of TDs and the circle highlights a group of closely spaced TDs.

apart. The average TD density for the sample was estimated to be $(5.1 \pm 0.4) \times 10^{8} \mathrm{~cm}^{-2}$ by averaging the dislocation count from a number of ECC images, including the one shown in Figure 2. The distribution of the dislocations was analyzed using $L(r)$, the deviation in the radial distribution compared with Ripley's $K$ function (Ripley, 1977) for randomly distributed points. $K(r)$ is the average number of other dislocations within a distance $r$ of each dislocation in the image (including a correction for dislocations close to the edge of the image). Our analysis reveals that there is a statistically significant clustering of dislocations compared to a random distribution, with a better than $90 \%$ confidence, for length scales $>500 \mathrm{~nm}$ (based on data for 150 $\mathrm{nm} \leq r \leq 6 \mu \mathrm{m})$. This may be because of the formation of lines of TDs; for example, the line of dislocations highlighted by the white ellipse in Figure 2. It has been shown that edge TDs may move during growth following coalescence, forming stable low-energy lines of dislocations preferentially aligned along the $\langle 11-20\rangle$ directions (Moram et al., 

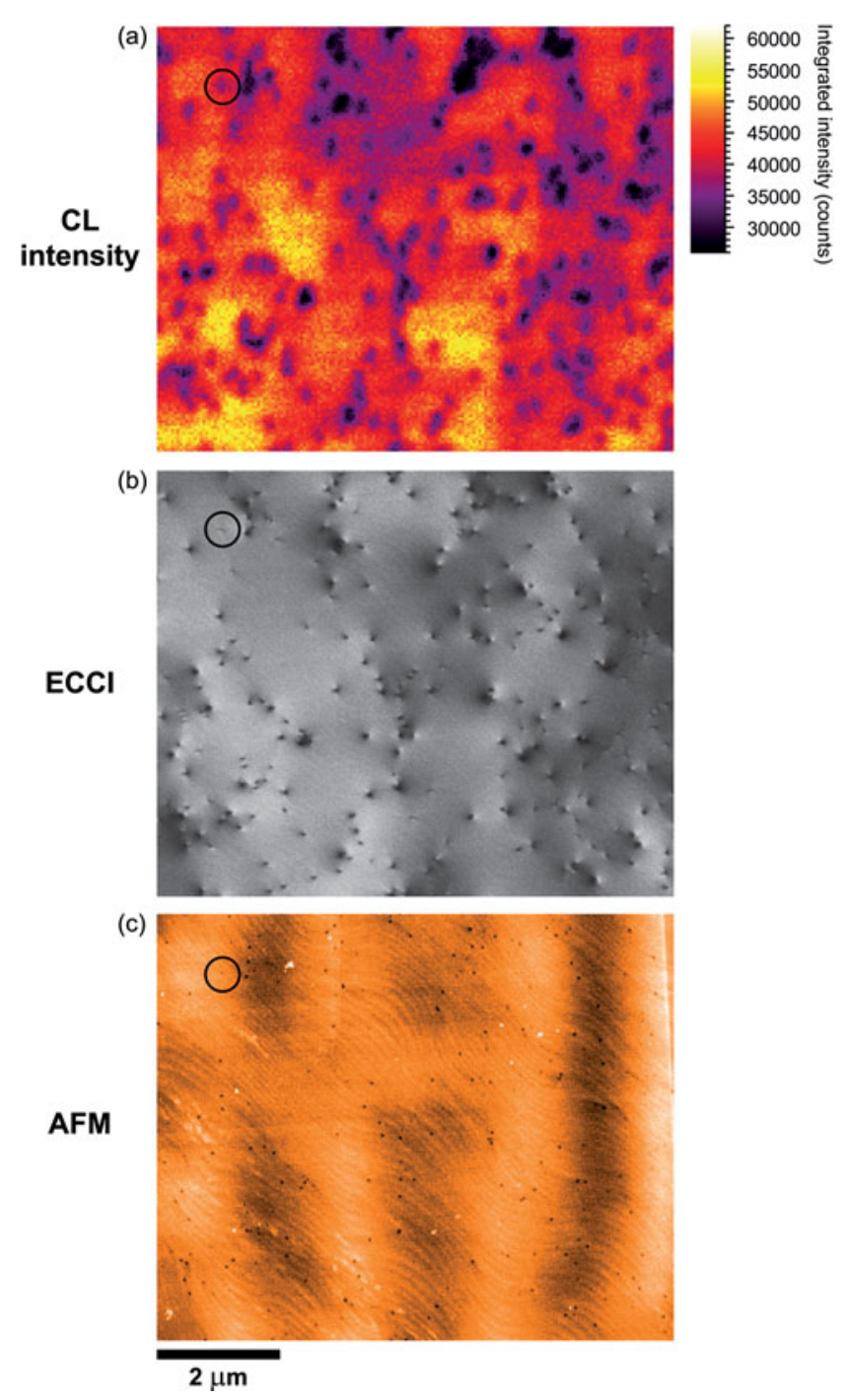

Figure 3. (a) Cathodoluminescence intensity map, (b) electron channeling contrast image, and (c) atomic force microscopy image of the GaN:Si thin film. The feature highlighted by the black circle is probably an inclined dislocation.

2009). In addition, there appear to be small groups of closely spaced dislocations; for example, the closely spaced dislocations highlighted by the white circle in Figure 2, which may be formed when large islands overgrow small, highly defective islands (Oliver et al., 2006a). A discussion of the application of several radial distribution functions to the analysis of the spatial distribution of dislocations in nitride thin films can be found in Moram et al. $(2009,2010)$.

Relative percentages of the different dislocation types were determined using the technique outlined in the "Materials and Methods" section (and described in detail in Naresh-Kumar et al., 2012a). Around $60 \%$ of the observed TDs are edge, $<2 \%$ are pure screw dislocations and the remaining are mixed type TDs.

Figure 3 shows (a) a CL intensity map, (b) an ECC image, and (c) an AFM image of exactly the same micronscale region of the Si-doped $c$-plane GaN thin film. These $8.5 \times 7 \mu \mathrm{m}$ images are cropped from a larger image of $16 \times$

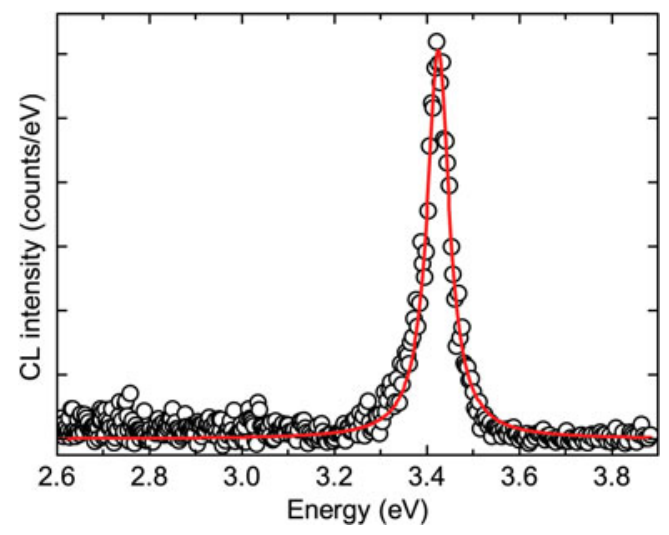

Figure 4. Typical cathodoluminescence spectrum from the GaN: Si thin film showing the experimentally acquired spectrum (circles) and the Voigt fit (line).

$13 \mu \mathrm{m}$ in size. The smaller images are presented here to allow the detail in the images to be examined and to allow easier comparison. Figure $3 \mathrm{a}$ is a CL map of the integrated intensity as determined from the area under the Voigt function fitted to each CL spectrum, an example of which is shown in Figure 4. The CL map was acquired following the acquisition of the ECC image.

For the ECC image shown in Figure 3b, it is possible to discriminate between TDs, which are more than $\approx 80 \mathrm{~nm}$ apart. A change in the channeling condition resulted in improved resolution when compared with Figure 2. Some of the TDs (of order 5\%) in the ECC image, such as the one highlighted by a black circle, are observed to have fainter and elongated contrast, that is, appear as short lines $\approx 100 \mathrm{~nm}$ in length. We surmise that these are projections of inclined TDs [TDs are observed to bend in Si-doped GaN (Brunner et al., 2012)]. Further work acquiring ECC images under different channeling conditions together with dynamical simulations of TD contrast are required to confirm this supposition.

For the CL map shown in Figure 3a, dark spots corresponding to single, isolated $(>400 \mathrm{~nm}$ from its nearest neighbors) TDs have a diameter of $\approx 150 \mathrm{~nm}$. In this case the size of the dark spots is dominated by the excitation volume. The larger dark spots correspond to clusters of TDs where the TDs are $<400 \mathrm{~nm}$ apart. The full ECC image revealed $\approx 750$ TDs; of order 400 of these are resolvable as single dislocations in the CL intensity map. A comparison of the ECC image and the CL intensity map shows a one-to-one correlation for these TDs. The regions of the sample showing highest CL intensity appear to coincide with regions of the sample free of dislocations. However, not all dislocation-free regions show bright CL, possibly indicating the presence of other defects such as point defects.

Acquiring an AFM image from the same region of the sample for which both the ECC image and CL map had been acquired (Fig. 3c) confirms that all dislocations are clearly visible for the channeling conditions selected for the acquisition of the ECC image. In this case the AFM was used to identify those dislocations that are of edge type and those with a screw component (i.e., screw and mixed dislo- 
cations) and it was not possible to differentiate between screw and mixed dislocations. The ratio of edge dislocations to dislocations with a screw component obtained from AFM is comparable to that obtained from the full ECCI analysis of a neighboring region of the sample. The AFM image also clearly shows atomic steps across the sample. Similar contrast is seen in the ECC image, where atomic steps are observed in the ECC image because of lattice distortion at the steps. Comparison of the ECC and AFM images showed that the inclined dislocations observed in the ECC image correspond to those identified as edge type by AFM, that is, their Burgers vector is the same as that for an edge dislocation, that is, $1 / 3[11-20]$.

Comparison of the CL map and AFM image reveals that the isolated, individual dark spots in the CL map corresponded to TDs of both edge and screw/mixed character. Thus, one may conclude that pure edge dislocations and dislocations with a screw component act as nonradiative recombination centers for our sample. No correlation was found between dislocation types and the size or intensity of the dark spots as seen in the CL intensity map. No definitive conclusions can be made with regard to the influence of pure screw dislocations on the luminescence for this sample because of the clustering of the dislocations and the small number of screw dislocations present in this sample. Future work on samples with a random dislocation distribution and a higher percentage of pure screw dislocations should enable us to elucidate the influence of pure screw dislocations on light emission from nitride semiconductors.

\section{Summary And Conclusions}

We have shown that it is possible to obtain useful and complementary data on the structural and luminescence properties of $\mathrm{GaN}$ thin films by obtaining AFM, ECC, and $\mathrm{CL}$ data from the same region of the sample. By comparing AFM and ECC images with a CL image from exactly the same micron-scale region of a sample, we have observed a one-to-one correlation between the presence of single TDs and dark spots resolved in the CL image. In addition, our results show that both pure edge dislocations and those with a screw component act as nonradiative recombination centers for the Si-doped $c$-plane $\mathrm{GaN}$ thin film investigated.

\section{ACKNOWLEDGMENTS}

This work was carried out with the partial financial support of the EU under the ITN RAINBOW (http://rainbow .ensicaen.fr/), grant agreement No: PITN-GA-2008-213238, EPSRC grant No: EP/D058686/1, and the University of Strathclyde.

\section{REFERENCES}

Albrecht, M., Weyher, J.L., LuczniK, B., Grzegory, I. \& PoRowski, S. (2008). Nonradiative recombination at threading dislocations in n-type GaN: Studied by cathodoluminescence and defect selective etching. Appl Phys Lett 92, 231909-1231909-3.

Amano, H., Miyazaki, A., Iida, K., Kawashima, T., Iwaya, M., Kamiyama, S., Akasaki, I., Liu, R., Bell, A., Ponce, F.A., Sahonta, S. \& Cherns, D. (2004). Defect and stress control of AlGaN for fabrication of high performance UV light emitters. Physica Status Solidi A 201, 2679-2685.

Bakshi, S.D., Sumner, J., Kappers, M. \& Oliver, R. (2009). The influence of coalescence time on unintentional doping in GaN/ sapphire. J Cryst Growth 311, 232-237.

Ban, K., Yamamoto, J., Takeda, K., Ide, K., Iwaya, M., Takeuchi, T., Kamiyama, S., Akasaki, I. \& Amano, H. (2011). Internal quantum efficiency of whole-composition-range AlGaN multiquantum wells. Appl Phys Express 4, 052101-1-052101-3.

Bruckbauer, J., Edwards, P.R., Wang, T. \& Martin, R.W. (2011). High resolution cathodoluminescence hyperspectral imaging of surface features in In GaN/GaN multiple quantum well structures. Appl Phys Lett 98, 141908-1-141908-3.

Brunner, F., Mogilatenko, A., Knauer, A., Weyers, M. \& ZetTLER, J.T. (2012). Analysis of doping induced wafer bow during GaN:Si growth on sapphire. J Appl Phys 112, 033503-1-033503-5.

Cao, X.A., LeBoeuf, S.F., D’Evelyn, M.P., Arthur, S.D., KretchMER, J., YAN, C.H. \& YANG, Z.H. (2004). Blue and near-ultraviolet light-emitting diodes on free-standing $\mathrm{GaN}$ substrates. Appl Phys Lett 84, 4313-4315.

DaI, Q., Schubert, M.F., Kim, M.H., Kim, J.K., Schubert, E.F., Koleske, D.D., Crawford, M.H., Lee, S.R., Fischer, A.J., Thaler, G. \& Banas, M.A. (2009). Internal quantum efficiency and nonradiative recombination coefficient of GaInN/GaN multiple quantum wells with different dislocation densities. Appl Phys Lett 94, 111109-1-111109-3.

Drouin, D., Couture, A.R., Joly, D., Tastei, X., Aimez, V. \& Gauvin, R. (2007). CASINO V2.42-A fast and easy-to-use modeling tool for scanning electron microscopy and microanalysis users. Scanning 29, 92-101.

Edwards, P.R., Jagadamma, L.K., Bruckbauer, J., Liu, C., Shields, P., Allsopp, D., Wang, T. \& Martin, R.W. (2012). Highresolution cathodoluminescence hyperspectral imaging of nitride nanostructures. Microsc Microanal 18, 1212-1219.

EdWARDS, P.R. \& Martin, R.W. (2011). Cathodoluminescence nano-characterization of semiconductors. Semicond Sci Technol 26, 064005-1-064005-8.

Elsner, J., Jones, R., Sitch, P.K., Porezag, V.D., Elstner, M., Frauenheim, T., Heggie, M.I., Öberg, S. \& Briddon, P.R. (1997). Theory of threading edge and screw dislocations in GaN. Phys Rev Lett 79, 3672-3675.

Harada, Y., Hikosaka, T., Kimura, S., Sugai, M., Nago, H., Tachibana, K., Sugiyama, N. \& Nunoue, S. (2012). Effect of dislocation density on efficiency curves in InGaN/GaN multiple quantum well light-emitting diodes. Proc of SPIE 8278, 82780J-1-82780J-6.

Karpov, S.Y. \& Makarov, Y.N. (2002). Dislocation effect on light emission efficiency in gallium nitride. Appl Phys Lett 81, 4721-4723.

Kneissl, M., Kolbe, T., Chua, C., Kueller, V., Lobo, N., StellMach, J., Knauer, A., Rodriguez, H., Einfeldt, S., Yang, Z., Johnson, N.M. \& Weyers, M. (2011). Advances in group IIInitride-based deep UV light-emitting diode technology. Semicond Sci Technol 26, 014036-1-014036-6.

Lester, S.D., Ponce, F.A., Craford, M.G. \& Steigerwald, D.A. (1995). High dislocation densities in high efficiency GaN-based light-emitting diodes. Appl Phys Lett 66, 1249-1251. 
Leung, K., Wright, A.F. \& Stechel, E.B. (1999). Charge accumulation at a threading edge dislocation in gallium nitride. Appl Phys Lett 74, 2495-2497.

Lymperakis, L., Neugebauer, J., Albrecht, M., Remmele, T. \& STrunk, H.P. (2004). Strain induced deep electronic states around threading dislocations in GaN. Phys Rev Lett 93, 196401-1-196401-4.

Martin, R.W., Edwards, P.R., O’Donnell, K.P., Dawson, M.D., Jeon, C.W., Liu, C., Rice, G.R. \& Watson, I.M. (2004). Cathodoluminescence spectral mapping of III-nitride structures. Physica Status Solidi A 201, 665-672.

Moram, M.A., Gabbai, U.E., Sadler, T.C., Kappers, M.J. \& OliVER, R.A. (2010). The use of spatial analysis techniques in defect and nanostructure studies. J Elect Mat 39, 656-662.

Moram, M.A., Oliver, R.A., Kappers, M.J. \& Humphreys, C.J. (2009). The spatial distribution of threading dislocations in gallium nitride films. Adv Mater 21, 3941-3944.

Naresh-Kumar, G., Hourahine, B., Edwards, P.R., Day, A.P., Winkelmann, A., Wilkinson, A.J., Parbrook, P.J., England, G. \& Trager-Cowan, C. (2012a). Rapid nondestructive analysis of threading dislocations in wurtzite materials using the scanning electron microscope. Phys Rev Lett 108, 135503-1135503-5.

Naresh-Kumar, G., Hourahine, B., Vilalta-Clemente, A., Ruterana, P., Gamarra, P., Lacam, C., Tordjman, M., DiForte-Poisson, M.A., Parbrook, P.J., Day, A.P., England, G. \& Trager-CowAN, C. (2012b). Imaging and identifying defects in nitride semiconductor thin films using a scanning electron microscope. Physica Status Solidi A 209, 424-426.

Norman, C.E. (2000). Challenging the spatial resolution limits of CL and EBIC. Solid State Phenom 78-79, 19-28.

Northrup, J.E. (2001). Screw dislocations in GaN: The Ga-filled core model. Appl Phys Lett 78, 2288-2290.

Northrup, J.E. (2002). Theory of intrinsic and H-passivated screw dislocations in GaN. Phys Rev B 66, 045204-1-045204-5.

Oliver, R.A., Kappers, M.J. \& Humphreys, C.J. (2006a). Insights into the origin of threading dislocations in $\mathrm{GaN} / \mathrm{Al}_{2} \mathrm{O}_{3}$ from atomic force microscopy. Appl Phys Lett 89, 011914-1-011914-3.

Oliver, R.A., Kappers, M.J., Sumner, J.S., Datta, R. \& HumPhreys, C.J. (2006b). Highlighting threading dislocations in
MOVPE-grown GaN using an in situ treatment with $\mathrm{SiH}_{4}$ and $\mathrm{NH}_{3}$. J Cryst Growth 289, 506-514.

Parish, C.M. \& Russell, P.E. (2007). Scanning cathodoluminescence microscopy. In Advances in Imaging and Electron Physics, Hawkes, P.W. (Ed.), pp. 2-115. San Diego, London: Academic Press.

Picard, Y.N., Caldwell, J.D., Twigg, M.E., Eddy, C.R., Jr., Mastro, M.A., Henry, R.L., Holm, R.T., Neudeck, P.G., TruneK, A.J. \& Powell, J.A. (2007). Nondestructive analysis of threading dislocations in $\mathrm{GaN}$ by electron channeling contrast imaging. Appl Phys Lett 91, 094106-1-094106-3.

Ripley, B.D. (1977). Modelling spatial patterns. J Roy Statist Soc Ser B 39, 172-212.

Schiavon, D., Binder, M., Peter, M., Galler, B., Drechsel, P. \& Scholz, F. (2013). Wavelength-dependent determination of the recombination rate coefficients in single-quantum-well GaInN/GaN light emitting diodes. Physica Status Solidi B 250, 283-290.

Trager-Cowan, C., Sweeney, F., Trimby, P.W., Day, A.P., Gholinia, A., Schmidt, N.H., Parbrook, P.J., Wilkinson, A.J. \& WATson, I.M. (2007). Electron backscatter diffraction and electron channeling contrast imaging of tilt and dislocations in nitride thin films. Phys Rev B 75, 085301-1-085301-8.

Wilkinson, A.J. \& Hirsch, P.B. (1997). Electron diffraction based techniques in scanning electron microscopy of bulk materials. Micron 28, 279-308.

Wright, A.F. \& Grossner, U. (1998). The effect of doping and growth stoichiometry on the core structure of a threading edge dislocation in GaN. Appl Phys Lett 73, 2751-2753.

Yacoвi, B.G. \& Holt, D.B. (1990). Cathodoluminescence Microscopy of Inorganic Solids. New York: Plenum Press.

YAKimov, E.B. (2012). Investigation of electrical and optical properties in semiconductor structures via SEM techniques with high spatial resolution. J Surf Invest-X-Ray 6, 887-889.

Yamamoto, N., Itoh, H., Grillo, V., Chichibu, S.F., Keller, S., Speck, J.S., DenBaArs, S.P., Mishra, U.K., Nakamura, S. \& Salviati, G. (2003). Cathodoluminescence characterization of dislocations in gallium nitride using a transmission electron microscope. J App Phys 94, 4315-4319. 